

\title{
An Event-Based Approach for Discovering Activities of Daily Living by Hidden Markov Models
}

K Viard, Mp Fanti, G Faraut, J-J Lesage

\section{To cite this version:}

K Viard, Mp Fanti, G Faraut, J-J Lesage. An Event-Based Approach for Discovering Activities of Daily Living by Hidden Markov Models. 15th IEEE Int. Conf. on Ubiquitous Computing and Communications, (IUCC 2016), Dec 2016, Granada, Spain. pp.85-92. hal-01378583

\section{HAL Id: hal-01378583 \\ https://hal.science/hal-01378583}

Submitted on 10 Oct 2016

HAL is a multi-disciplinary open access archive for the deposit and dissemination of scientific research documents, whether they are published or not. The documents may come from teaching and research institutions in France or abroad, or from public or private research centers.
L'archive ouverte pluridisciplinaire HAL, est destinée au dépôt et à la diffusion de documents scientifiques de niveau recherche, publiés ou non, émanant des établissements d'enseignement et de recherche français ou étrangers, des laboratoires publics ou privés. 


\title{
An Event-Based Approach for Discovering Activities of Daily Living by Hidden Markov Models
}

\author{
K. Viard ${ }^{1,2}$, MP. Fanti ${ }^{2}$, G. Faraut ${ }^{1}$, J-J. Lesage ${ }^{1}$ \\ ${ }^{1}$ LURPA, ENS Cachan, Univ Paris Sud, Université Paris-Saclay \\ F-94235 Cachan, France \\ \{kevin.viard; gregory.faraut; jean-jacques.lesage\} @ens-cachan.fr \\ ${ }^{2}$ Department of Electrical and Information Engineering, Polytechnic of Bari, \\ Via Orabona 4, 70125 Bari, Italy \\ mariapia.fanti@poliba.it
}

\begin{abstract}
Smart Home technologies may improve the comfort and the safety of frail people into their home. To achieve this goal, models of Activities of Daily Living (ADL) are often used to detect dangerous situations or behavioral changes in the habits of these persons. In this paper, an approach is proposed to build a model of ADLs, under the form of Hidden Markov Models (HMMs), from a training database of observed events emitted by binary sensors. The main advantage of our approach is that no knowledge of actions really performed during the learning period is required. Finally, we apply our approach to a real case study and we discuss the quality of the results obtained.
\end{abstract}

Keywords-Smart Home, Activity Discovery, Hidden Markov Model

\section{INTRODUCTION}

Life expectancy has continuously increased in industrialised countries over the last decades. According to recent studies $[1,2]$, the part of the population composed of dependant or aged people is expected to rise continuously until 2060 and the part of people aged 60 or over will reach $30 \%$ in many countries in 2050 . It is therefore very important to propose new technological and social frameworks allowing aged or disabled people to live autonomously in a safe environment. Smart homes and Ambient Assisted Living (AAL) technologies [3, 4] are now mature enough for allowing the deployment of health monitoring of frail persons at home, ensuring a continuum of care from hospital to home. The modelling of Activities of Daily Living (ADL) and the detection of their drifts is of major interest in ensuring health monitoring. Indeed, evolution or deviations of ADL are often symptoms of pathologies frequently encountered for aged people. It is therefore necessary to be able to build relevant models of activities and habits of life of the person to be monitored.

Different sensor technologies are used in the field of AAL. They are more or less intrusive, they provide more or less semantically rich information and are more or less expensive $[11,12,14]$. In order to help the user to accept the observation of his movements and to guarantee the respect of his privacy we chose to use non-wearable and non-intrusive sensors even if this makes multi-habitant issue harder to solve
[5]. Furthermore, to reduce the costs (aging of population is a mass problem), we favour low-cost sensors. Such sensors are mostly binary sensors (door barrier sensors, motion detectors) or sensors delivering a signal that can be interpreted as binary using a threshold (electricity consumption, water flow or pressure sensor for instance) [5]. The resulting problem for $\mathrm{ADL}$ is therefore to build semantically rich models allowing to know in real time which activity is performed by an inhabitant living in a smart home, from semantically poor sequences of observed binary events.

Models for ADL modelling can be deterministic [6, 7] or probabilistic [8,9]. Because human behaviour is of course non-deterministic and can even become irrational in case of patients suffering of specific pathologies, probabilistic models offer a good modelling framework for ADL, and especially Hidden Markov Models (HMM) [10]. Nevertheless, despite numerous strong results obtained by the use of HMM for modelling ADLs [11, 9], the building method of the Hidden Chain is never given or seems to not be a topic of interest.

In particular, the paper focuses on the activities discovery problem, i.e., the issue of modelling the activities performed by the inhabitants by studying the recorded database of signals emitted by binary sensors. To this aim, we propose a general technic devoted to the automatic specification of a HMM that is able to cope with the non-determinism of the human behaviour at home.

A brief review of related works is proposed in the following section. In Section III, the problem statement as well as basic recalls on HMM are given. In Section IV, the procedure to automatically build an activity model is presented. This method is illustrated in Section $\mathrm{V}$ on a real example extracted from literature. In the last section, the contribution is summarized and an outlook of future work is given.

\section{RELATED WORKS}

Three points of view about ADL modelling can be of interest with the aim of monitoring the health of elderly and disable people: the objectives of ADL modelling, the chosen sensors technologies and the formalism describing the model. 


\section{A. Objectives of ADL modelling}

Research works in the field of ADLs can be decomposed into four main topics [11, 12, 8, 13]: Activities Discovery (AD), Activities Recognition (AR), Detection of Deviations (DD) and Activities Prediction (AP).

The goal of $\mathrm{AD}[11,12]$ is to generate one or several models of activities by studying recorded learning database. The models can be built by an expert or they can be built automatically from sensor signals database. AD is performed off-line and mainly depends on the quantity and the quality of available information.

The goal of AR [11,8], which is computed online, is to detect which activity is performed by the person using template models previously built in AD step.

$\mathrm{DD}$ and $\mathrm{AP}$ are steps that follow $\mathrm{AD}$ and $\mathrm{AR}$ [11]. Indeed, once an activity is detected, the aim of DD is to determine if there is a deviation from the habitual behaviour by taking into account the activities models built in AD. Considering AP, its aim is to determine the next activity that should be performed by using past events and the current detected activity. The links between these different tasks are illustrated in Fig. 1.

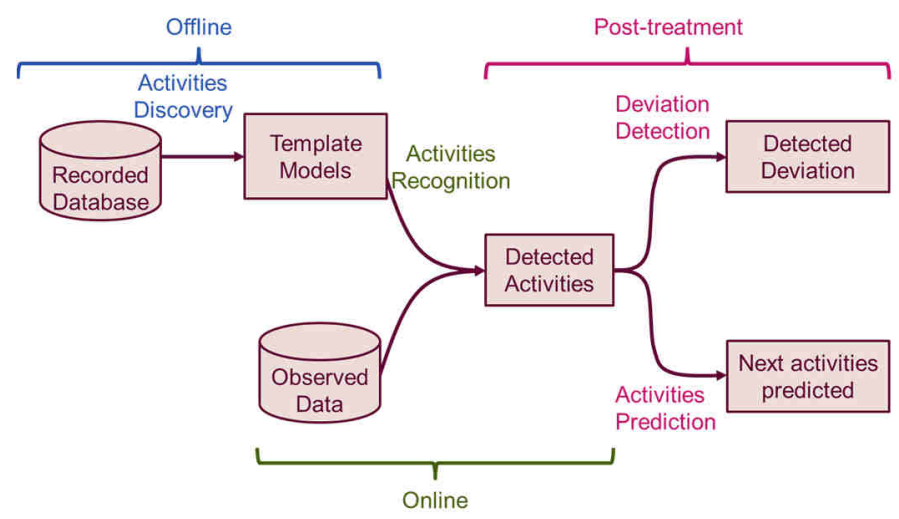

Fig. 1. Missions of assistance and monitoring of ADLs

In the sequel of the paper, we are concentrate on $\mathrm{AD}$ step only.

\section{B. Sensors Technologies}

Sensor choices have a great influence on the ADL models because they define the type of observed information, which can be used for building AD models.

Sensors can be classified using different methods and criteria. In the related literature [14], sensors are mainly split into non-intrusive or intrusive sensors. Intrusive sensors, like cameras, microphones, etc. are interesting because the information they provide is semantically rich and allows to easily perform AD. Nevertheless, even if academic works with intrusive sensors are numerous, experimental works are not: privacy is important and people are not pleased to feeling observed by cameras. Non-intrusive sensors like binary or wearable sensors are well accepted because people do not feel observed. However, wearable sensors require that person pay attention to not forgot to wear it $[11,12]$.

These are reasons why we chose to use only non-wearable binary sensors. Indeed, information given by this kind of sensors does not depend on the ability or the willingness of the inhabitant to provide this information.
Nevertheless, information provided by binary sensors are semantically poor, what makes difficult to infer a model of the observed behaviour [5].

\section{Modelling Formalism}

Human behaviour is typically not deterministic, unpredictable and can even be irrational. Hence, it is necessary to choose modelling formalism allowing coping with all these characteristics.

Among all papers dealing with $\mathrm{AD}$, different kinds of models representing activities can be found.

In $[6,15]$, the authors detect recurrent patterns in numerous event sequences. They obtain a list of specific sequences traducing the existing routines in a registered database. Even if those routines are semantically close to recurring actions, it is difficult to link them to an a priori known action such as "dressing" or "bathing". Moreover, pattern-mining algorithms set out in those papers are, by definition, not robust for non-deterministic behaviour. With the same kind of limits, the authors of [7] obtain activity models as a list of detectable actions analysing people poses by cameras. In [12], authors present a survey on AR taking different types of models as input (sequence of sensors signals, Markov models, poses detection and Gaussian laws) but using only wearable sensors. In [8] a new extension of HMM is proposed: the Switching Hidden Semi-Markov Model (S-HSMM) generated using cameras output.

In most of these papers a deterministic model of the observed behaviour is provided or obtained from information given by intrusive sensors. Nevertheless, some works propose to build models from binary sensors only. In [6], authors build automata representing performed activities. However, the least difference in the observed behaviour leads to the impossibility to recognise the current activity. In $[11,9]$, authors present a method expressing activities as HMM and using binary sensors information. In their studies, authors generate HMM by the use of learning data composed by the following elements: i) log of sensor events, ii) log of action realised at the same time and iii) a description of each activity as a set of actions. However, in [16], Tapia et al. explain the difficulty to obtain such a log of occurred actions that is precise, complete and true. The use of uncertain data for generating standard activity model can lead to incoherent or false models. This is the reason why in this paper, we propose an approach allowing to build activity models from the observed behaviour of the inhabitant only, without needing to know the actually actions performed.

In this paper, we focus on $\mathrm{AD}$; the goal is to automatically build activities models. To respect privacy without requiring willingness of the inhabitant, only binary sensors are considered. To be able to represent the non-determinism of human behaviour, we model activities in the HMM framework. The procedure we propose is generic and only based on the knowledge of home topology and on a record of observed binary sensor events.

\section{PRoblem Statement And Hidden Markov Model}

\section{A. Activity of Daily Living}

According to [17], ADL is defined by the medical community as "the things we normally do in daily living including any daily activity we perform for self-care, work, homemaking, and leisure". 
This definition can be completed by the semantic classification presented in $[7,11]$ describing an activity as a set of actions that can be see as a set of elementary moves. For instance, the activity "taking care of personal hygiene" is composed of the actions "taking a bath, toileting, washing hands..." and each action can be composed of moves "go on the bath, open the bath hot faucet, open the bath cold faucet...".

By using binary sensors, we are only able to capture information linked to elementary moves. Hence, our semantic classification is summarised in Fig. 2.

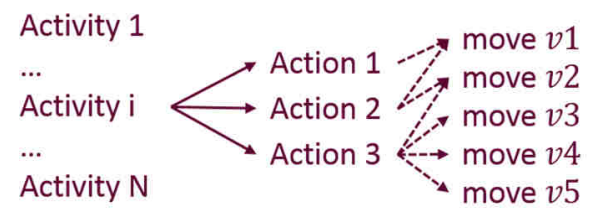

Fig. 2. Semantic classification between activities, actions and moves.

\section{B. Problem Definition}

Existing methods to generate HMM using registered data also need the actual description of actions really performed during the data record. Several approaches are used for obtaining this additional information.

First, the observed person can be asked to describe continuously each time what he/she is performing. This approach supposes that an efficient system is developed to let the person declare actions without influencing his daily living. Moreover, the ability and the willingness of the patient to declare his activity has a huge impact on the result and false declaration may lead to a bad conclusion. Therefore, this approach is not available for patient suffering for certain pathologies.

Second, an expert, mainly a therapist, can interpret recorded data. This possibility assumes that the expert is able to treat at least fifteen days of sensor event logs and to conclude what a person is doing at each moment. Even if for practical reasons it is possible to add cameras during the learning phase, it remain difficult to be sure that the expert do not make omission or mistakes. Furthermore, considering the duration of this analysis, it is undeniably too expensive.

In this work, we prefer not to use recorded played actions because it is difficult to obtain reliable information whatever approach is employed. Therefore, the problem is to reconstruct this information by using only a detailed description of the smart home equipped with the suitable number of sensors, the recorded sensor outputs and the relationship among activities and actions. Fig. 3 reports a scheme of the activity discovery considered in the paper.

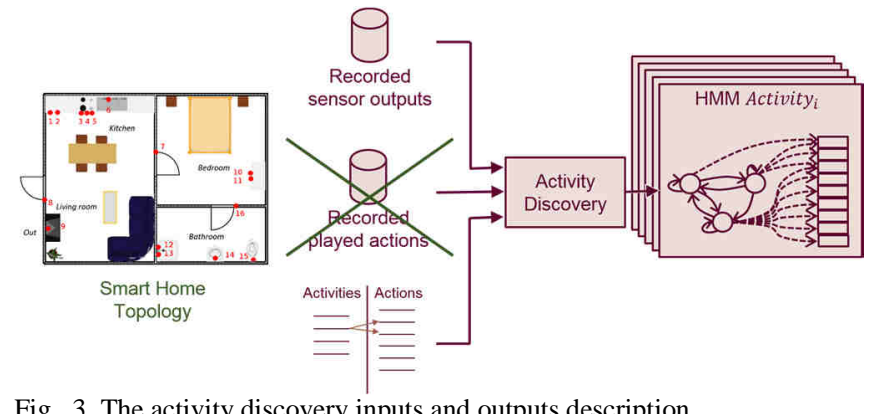

Fig. 3. The activity discovery inputs and outputs description.

\section{HMM Basis and Notations}

Markov models are observable "since output of the process is the set of states at each instant time, where each state corresponds to a physical event" [10]. Moreover, "HMM is an extension of Markov models: it is a doubly embedded stochastic process with an underlying process that is not observable (it is hidden), but can only be observed through another set of stochastic processes that produce the sequence of observation".

HMM can be represented by the 5-uplet $\lambda=(S, V, A, B, \pi)$ where:

$S=\left\{s_{1}, s_{2} \ldots S_{|S|}\right\}$ is the set of hidden states; the active state at the time $t$ is denoted $q_{t}$;

$V=\left\{v_{1}, v_{2} \ldots v_{|V|}\right\}$ is the set of observable events;

$A=\left\{a_{i, j}\right\}$ is the set of transition probability distribution where

$$
a_{i, j}=P\left[q_{t+1}=s_{j} \mid q_{t}=s_{i}\right], \quad i, j \in[1,|S|]
$$

$B=\left\{b_{h}(k)\right\}$ is the set of observation event probability distribution in state $\mathrm{h}$ :

$$
b_{h}(k)=P\left[v_{k} \text { at } t \mid q_{t}=s_{h}\right], h \in[1,|S|], k \in[1,|V|],
$$
$\pi=\left\{\pi_{1}, \pi_{2} \ldots \pi_{|S|}\right\}$ is the set of state initial probabilities:

$$
\pi_{l}=P\left[q_{1}=s_{l}\right], \quad l \in[1,|S|]
$$

Fig. 4. proposes a graphical representation of a HMM with 3 hidden states and 5 observable events. Transitions between hidden states are denoted by solid arcs and links between states and observable events are described by dotted arcs.

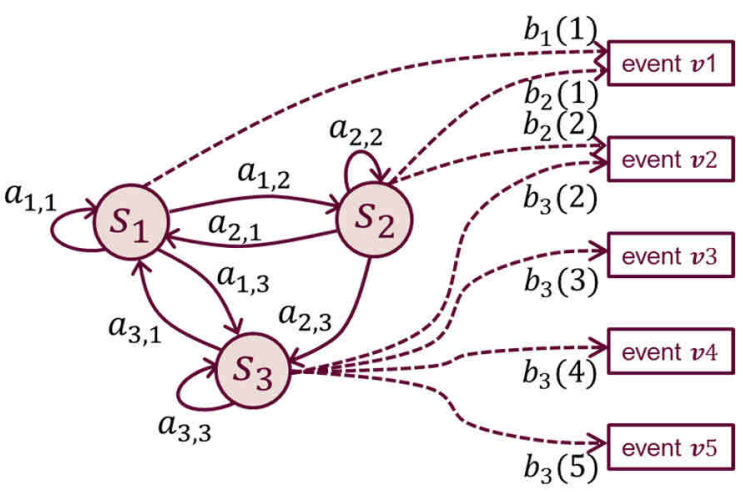

Fig. 4. Basic representation of HMM

In HMMs, events cannot occur simultaneously. This assumption is not a limitation for ADL modelling because it is always possible to distinguish very time close events in smart homes.

\section{BUILDING ACTIVITY MODELS BY HMM}

The procedure we propose to build the HMM representing the activity from the topology of the smart home and their decomposition in actions, can be decomposed in four steps: i) association of relevant sensors to actions, ii) generation of HMM structure, iii) database of event logs exploration for iv) probabilities computation.

\section{Definition 1:}

Each Activity $A c t_{i}$ is modelled by a HMM $\lambda_{A c t_{i}}$ represented by a 5 -uplet $\lambda_{A c t_{i}}=(S, V, A, B, \pi)$ where:

- each hidden state $s_{i} \in S$ represents an action composing this activity,

- $\quad$ each observable event $v_{j} \in V$ represents a change of value of a sensor signal. 


\section{Procedure:}

Step 1 - Association of relevant sensors to actions

For given links between activities and actions, an expert has to link each action to relevant sensors (sensors whose events reveal this action execution) as shown in Fig. 5.This can be done using the location of sensors in the dwelling, in relation to the activity. For instance, a sensor in the kitchen could be linked to the action "prepare meals" but cannot be linked to the action "have a shower". Furthermore, a sensor can be linked to several actions and an action can be linked to more than one sensor.

\section{Step 2 - Generation of HMM structure:}

A HMM structure is automatically generated for each activity. For that, one hidden state is created for each action composing this activity. At this step, all transitions between all states are created allowing to reproduce the execution of any sequence of actions (transitions with an associated probability equal to 0 will be removed at step 4). The relevant output events and their links with actions are added in accordance with the result of the previous step. At this point, the structure of the HMM is fully built. The probabilities associated to transitions are now to be computed. A graphical representation of the result of the two first steps is given on Fig. 5 .

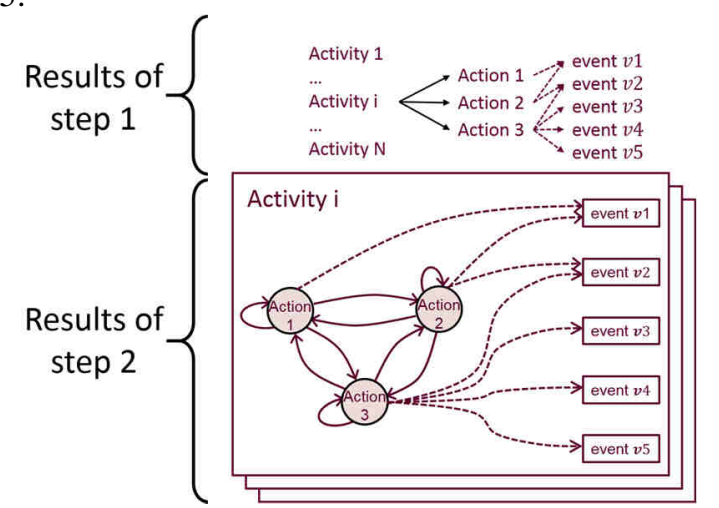

Fig. 5. Activities/action/events links and generation of model structures

\section{Step 3 - Database of event logs exploration}

The database consists in long sequences of recorded events in which we have to look for local succession of actions. To do that, we can explore these sequences by considering a sliding subsequence of events only.

In [18], authors present three common approaches for processing such subsequence of events: i) explicit segmentation, ii) time based windowing and iii) sensor event based windowing (see Fig. 6).



Fig. 6. Common approaches for processing streaming data (from [18])

In this paper, sensor event based windowing is chosen because this technic is more efficient for timely closed actions than explicit segmentation and more adapted to discrete time than time based windowing.

The principle of this approach is to observe a large sequence, here the complete database, by using a window of length $l$ events, and by sliding this window along the sequence as shown in Fig. 7. At each step, a sequence $\sigma_{w}$ is observed. For each activity $A c t_{i}$, sequence $\sigma_{w}$ is projected on the linked event set. Thus, for each activity and for each window, a projected sequence $\sigma_{p}$ is obtained.

Formally, let:

- $\quad \Sigma$ be the set of all possible sensor events in the smart home

- Action $_{h}$ be an action linked to the activity $A c t_{i}$,

- $V\left(\right.$ Action $\left._{h}\right)$ be the set of events linked to the Action $_{h}$,

- $V\left(A c t_{i}\right)$ the set of events linked to the activity $A c t_{i}$.

Remark: $V\left(\right.$ Act $\left._{i}\right)=\bigcup_{h} V\left(\right.$ Action $\left._{h}\right) \subseteq \Sigma$

The projection of a sequence $\sigma_{w} \in \Sigma^{*}$ on the set $V\left(A c t_{i}\right)$ is defined by:

with

$$
\operatorname{Proj}: \Sigma^{*} \rightarrow V\left(A c t_{i}\right)
$$

$$
\begin{gathered}
\operatorname{Proj}(\epsilon):=\epsilon \\
\operatorname{Proj}(v):=\left\{\begin{array}{l}
v \quad \text { if } v \in V\left(A_{c} t_{i}\right) \\
\epsilon \quad \text { if } v \in \Sigma \backslash V\left(A_{i}\right)
\end{array}\right) \\
\operatorname{Proj}(\sigma v):=\operatorname{Proj}(\sigma) \operatorname{Proj}(v) \text { for } \sigma \in \Sigma^{*}, v \in \Sigma
\end{gathered}
$$

Where $\Sigma^{*}$ is the infinite set of all possible sequences on $\Sigma$, and $\epsilon$ is the sequence of length 0 , i.e. the sequence empty of event.

Each time projections are determined, some indicators of frequency of occurrence of events are computed in order to evaluate probabilities that will be associated to HMMs (presented in step 4):

- $\quad N_{v_{j}}$ : number of times where event $v_{j}$ is observed in the projected sequence $\sigma_{p}$,

- $\quad N_{\text {init } v_{j}}$ : number of times where event $v_{j}$ is the first event of the projected sequence $\sigma_{p}$,

- $\quad N_{v_{j} \rightarrow v_{k}}$ : number of times where event $v_{k}$ follows event $v_{j}$ in the projected sequence $\sigma_{p}$.

Fig. 7 gives an example to illustrate the previously described operations with a sliding window of length 10 .

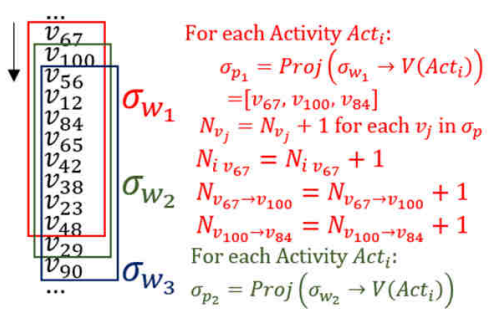

Fig. 7. Database exploration

Step 4 - Probabilities computation:

In the following the computation of probabilities in the HMM of each $A c t_{i}$ is described. 
The conditional probability $b_{h}(k)$ traducing the chance to observe $v_{k}$ considering all events of Action $_{h}$ is determined as follows:

$$
b_{h}(k)=P\left(v_{k} \text { at } t \mid q_{t}=\text { Action }_{h}\right) \quad \text { if } v_{k} \in V\left(\text { Action }_{h}\right)
$$

Moreover, the probability $\pi_{h}$ that Action $_{h}$ is initial in the Act $_{i}$ model is:

$$
\pi_{h}=P\left(q_{1}=\text { Action }_{h}\right)=\sum_{v_{k} \in V\left(\text { Action }_{h}\right)} \frac{1}{C_{v_{k}}} \times P\left(v_{k} \text { at } t=1\right)
$$

In (2), we consider an equi-probability of occurrence between actions linked to $v_{k}$ when it is observed as initial. Moreover, $C_{v_{k}}$ represents the number of states associated to $v_{k}$.

Finally, the conditional probabilities $a_{h, j}$ to observe event of Action $_{j}$ after having observed an event of Action $_{h}$ in the projected sequence $\sigma_{p}$ is determined as follows:

$$
\begin{aligned}
& \text { If } h \neq j, \\
& a_{h, j}=\sum_{v_{k} \in V\left(\text { Action }_{h}\right)} \sum_{\substack{v_{m} \in V\left(\text { Action }_{j}\right) \\
v_{m} \notin V\left(\text { Action }_{h}\right)}} \frac{1}{C_{v_{m}}} P\left(v_{m} \text { at } t+1 \mid v_{k} \text { at } t\right)
\end{aligned}
$$

otherwise:

$$
a_{h, h}=\sum_{v_{k} \in V\left(\text { Action }_{h}\right)} \sum_{v_{m} \in V\left(\text { Action }_{h}\right)} P\left(v_{m} \text { at } t+1 \mid v_{k} \text { at } t\right)
$$

In (3) and (4), it is considered that the succession of two events $v_{k}$ and $v_{m}$ linked to a same Action $_{h}$ cannot represent a transition from this Action $_{h}$ to an other Action $_{j}$ (event if $v_{m} \in$ Action $\left._{h}\right)$.

The discrete formulation of equations (1) to (4) using the previously defined variables are:

$$
\begin{aligned}
& b_{h}(k)=\frac{N_{v_{k}}}{\sum_{m \in\left(\text { Action }_{h}\right)} N_{v_{m}}} \quad \text { if } v_{k} \in V\left(\text { Action }_{h}\right) \\
& \pi_{h}=\sum_{v_{k} \in V\left(\text { Action }_{h}\right)} \frac{1}{C_{v_{k}}} \times \frac{N_{\text {init }_{k}}}{\sum_{v_{m} \in V\left(\text { Act }_{i}\right)} N_{\text {init }_{m}}}
\end{aligned}
$$

If $h \neq j:$

$$
a_{h, j}=\sum_{v_{k} \in V\left(\text { Action }_{h}\right)} \sum_{\begin{array}{c}
v_{m} \in V\left(\text { Action }_{j}\right) \\
v_{m} \notin V\left(\text { Action }_{h}\right)
\end{array}} \frac{1}{C_{v_{m}}} \frac{N_{v_{k} \rightarrow v_{m}}}{N_{V\left(\text { Action }_{h}\right) \rightarrow \cup_{n \neq h} V\left(\text { Action }_{n}\right)}}
$$

otherwise:

$$
a_{h, h}=\sum_{v_{k} \in V\left(\text { Action }_{h}\right)} \sum_{v_{m} \in V\left(\text { Action }_{h}\right)} \frac{N_{v_{k} \rightarrow v_{m}}}{N_{V\left(\text { Action }_{h}\right) \rightarrow V\left(\text { Action }_{h}\right)}}
$$

with $N_{V 1 \rightarrow V 2}=\sum_{v_{k} \in V 1} \sum_{v_{m} \in V 2} \quad N_{v_{k} \rightarrow v_{m}}$

At the end of the procedure, the HMM structure is completed and the probabilities are computed.

In order to illustrate the proposed method, next section presents an application to a real smart home.

\section{Case Study}

\section{A. Flat topology and recorded data}

Since we have not equipped dwelling in our disposition, we applied the proposed method to an existing database generated by Tapia et al. [16]. This database is corresponding in two week of observation of a single normal person living in his own dwelling equipped with 77 binary sensors. The topology of the flat, as well as the sensor placement, is shown on Fig. 8.



Fig. 8. Smart Home topology of the case study (the sensors used in the developed example are circled in blue).

For two weeks, the inhabitant lived normally at home and generated a database composed of 2323 lines (an event occurrence at each line), whose structure is presented in Fig. 9. We develop an experimental software, coded in python to automatically construct the HMMs starting from step 2. In the following example, the building of the HMMs took 1.4 seconds using a standard laptop with a processor frequency of $2.50 \mathrm{GHz}$.

\begin{tabular}{llll} 
Date & Hour & $\begin{array}{l}\text { Sensor } \\
\text { ID }\end{array}$ & $\begin{array}{l}\text { Sensor } \\
\text { Name }\end{array}$ \\
\hline $03 / 27 / 2003$ & $06: 43: 40$ & 67 & Cabinet \\
$03 / 27 / 2003$ & $06: 44: 06$ & 100 & Toilet flush \\
$03 / 27 / 2003$ & $06: 44: 20$ & 101 & Light switch \\
$03 / 27 / 2003$ & $06: 44: 35$ & 57 & Medecine cabinet \\
$03 / 27 / 2003$ & $06: 44: 36$ & 58 & Medecine cabinet \\
$03 / 27 / 2003$ & $06: 44: 49$ & 67 & Cabinet \\
$03 / 27 / 2003$ & $06: 45: 45$ & 82 & Drawer
\end{tabular}

Fig. 9. Extract of the input recorded data. 




Fig.. 11. Activity "Personal hygiene" modelled by an HMM

\section{B. Method Application}

Step 1 - Association of relevant sensors to actions

The input of this step is the knowledge of the links between activities and actions and the instrumentation of the flat (Fig. 8). Those links are partially shown in Fig. 10(a). For space reasons, we focus on "personal hygiene" activity only.

This step consists in associating actions to zones of the flat where they can be performed. The list of relevant sensors allowing to observe these actions are deduced (Fig. 10(b)).

From now, all steps of HMM building are automatically processed.

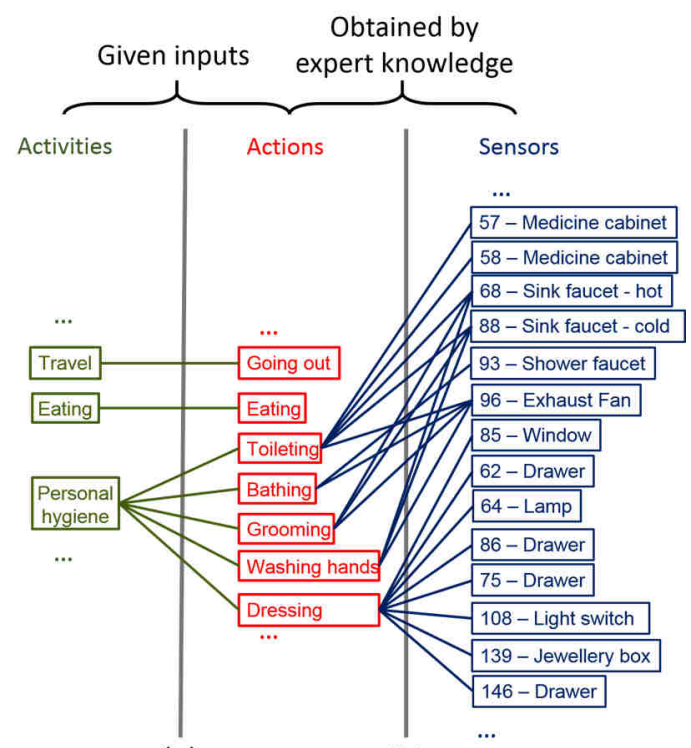

(a)

(b)

Fig. 10. Complete links between activities, actions and sensors.

\section{Step 2 - Generation of HMM structures}

In this step, for instance, the "Personal hygiene" activity is modelled by a HMM structure with 5 hidden states (one for each of the five actions that compose the activity), 14 observable events (the 14 sensors a priori involved in the activity "Personal hygiene" are encircle in Fig. 8), $5^{2}$ possible transitions between hidden states and 20 event emission transitions (the link between the state "dressing" and the event "85 - Window" was automatically deleted because this event has never been observed in logs).

\section{Step 3 - Database of event logs exploration}

Three different values of the length of the sliding window have been tested: 5, 10 and 20. It appears that a window with a length of 10 events is a good trade-off between observability of events and computational effort.

For each observed event in each sliding window, we calculate indicators presented in Section IV. For instance, for sensors $v_{57}, v_{58}$ and $v_{68}$ it holds:

$N_{v_{57}}=995, N_{v_{58}}=795, N_{v_{68}}=1504$,

$N_{\text {init } v_{57}}=214, \quad N_{\text {init } v_{58}}=183, N_{\text {init }} v_{68}=329$,

$N_{v_{57} \rightarrow v_{57}}=15, N_{v_{57} \rightarrow v_{58}}=186, N_{v_{57} \rightarrow v_{68}}=101$.

Step 4 - Probabilities computation:

The HMM probabilities are computed using formulas (5) to (8). Activities are now fully modelled and Fig. 11 illustrates the final HMM obtained for the activity "Personal hygiene". Transitions between hidden states associated to a probability equal to 0 have been removed because they correspond to non-observed succession of actions. In a same way, event emission transitions labelled with a null probability are symptomatic of mistakes in the installation of sensors broken or not used; they can be removed.

\section{Discussion}

The same approach as the one developed in the previous section has been used for building HMM models for the 15 activities, composed of 24 actions, described in [16].

In order to validate the accuracy of the proposed models, event sequences of the learning database was replayed on the constructed HMMs, what allowed to infer which actions was performed by the inhabitant. Afterwards, we compare these results to the declaration of actions done by the inhabitant during the learning period (even if it is mentioned in [16] that these declarations include error and omission). We report the outcome of these comparisons in the confusion matrix shown in Fig. 12, with:

$p_{l ; c}=P\left(\right.$ Action $_{l}$ detected $\mid$ Action $_{c}$ declared $)$

where $l$ and $c$ are respectively the indices of lines and the columns of the matrix. The matrix on Fig. 12 includes only 10 actions:

1- "Watching TV", 2- "Going out", 3- "Doing laundry", 4"Washing dishes", 5- "Preparing eat", 6- "Washing hands", 7- "Bathing", 8- "Dressing", 9- "Grooming", 10- "Toileting" 
and 11- "None" represents that no action was detected when one is declared.

\begin{tabular}{|c|c|c|c|c|c|c|c|c|c|c|c|}
\hline \multirow{11}{*}{ 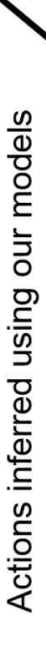 } & 1 & 100 & 0 & 0 & 0 & 0 & 0 & 0 & 0 & 0 & 0 \\
\hline & 2 & 0 & 100 & 0 & 0 & 0 & 0 & 0 & 0 & 0 & 0 \\
\hline & 3 & 0 & 0 & 100 & 50 & 0 & 0 & 0 & 0 & 0 & 0 \\
\hline & 4 & 0 & 0 & 91 & 50 & 0 & 0 & 0 & 0 & 0 & 0 \\
\hline & 5 & 0 & 0 & 0 & 0 & 85 & 0 & 0 & 0 & 0 & 0 \\
\hline & 6 & 0 & 0 & 0 & 0 & 0 & 0 & 0 & 0 & 0 & 0 \\
\hline & 7 & 0 & 0 & 0 & 0 & 0 & 0 & 31 & 0 & 0 & 4 \\
\hline & 8 & 0 & 0 & 0 & 0 & 0 & 0 & 0 & 44 & 8 & 4 \\
\hline & 9 & 0 & 0 & 0 & 0 & 0 & 0 & 0 & 0 & 0 & 0 \\
\hline & 10 & 0 & 0 & 0 & 17 & 0 & 0 & 46 & 50 & 42 & 64 \\
\hline & 11 & 0 & 0 & 0 & 33 & 15 & 100 & 23 & 6 & 50 & 28 \\
\hline
\end{tabular}

Fig. 12. Confusion matrix

Analysing this matrix, we can conclude that actions "Watching TV"(1), "Going out"(2) and "Preparing eat"(5) are perfectly inferred by the proposed approach.

On the other hands, actions "Doing laundry"(3) and "Washing dishes"(4) are non-dissociable. Indeed, these two actions are performed by using two different equipments (a washing machine and a dishwasher). Each one is equipped with vibration sensor to detect its operation but these two machines are placed one over the other. Therefore as soon as one machine is on operation, it is detected by one or sometime both sensors. This technological characteristic explains why in the confusion matrix, the couple \{action 3, action 4$\}$ is very well detected, even if it is not possible to distinguish which one of these two actions is actually performed.

The reason why "Bathing"(7), "Dressing"(8), "Grooming"(9) and "Toileting"(10) are linked is different. In fact, these actions are too close and have too much joint sensors what makes the differentiation harder. Moreover, the inhabitant can also have some trouble to dissociate actions like "Toileting"(10) and "Grooming"(9). In this case, the problem comes from the expert classification of actions.

The observation of "Toileting" when "Washing dishes" can be due a person mistake too. In [16] Tapia et al. precise that it is not possible to cancel an action (badly declared) in their declaration, even if the patient knows his mistake and wants to correct it. Despite these small differences, the results in the confusion matrix prove that the detected actions are close to the declared ones.

To conclude, the confusion matrix shows that we can build efficient models of habit without manually detecting realised actions during the learning phase.

\section{ACKNOWLEDGMENT}

The research of K. VIARD is supported by Université Franco Italienne, VINCI programme 2016.

\section{CONCLUSION}

In this paper, we propose an approach for Activity of Daily Living discovery. We develop a procedure to model activities by Hidden Markov Models based on the knowledge of a training event logs database, a given decomposition of activities into actions and the detailed description of the instrumentation of the smart home. The main advantage of the presented approach is that the knowledge of actions really performed during the learning period is not required. Finally, we applied the approach to a real case study and we discussed the quality of the obtained results.

Future works will be devoted to the use of HMM Activity models for the detection of deviations in the behaviour of people to be monitored.

\section{REFERENCES}

[1] E. Eurostat, "Population structure and ageing," 2010. Available online:

http://ec.europa.eu/eurostat/statisticsexplained/index.php/Population_structure_and_ageing

[2] W. H. Organization et al., "Good health adds life to years:

Global brief for world health day 2012," 2012.

Available online:

http://www.who.int/ageing/publications/whd2012_glob al_brief/en/

[3] P. Rashidi and A. Mihailidis, "A survey on ambientassisted living tools for older adults," IEEE Journal of Biomedical and Health Informatics, 17(3), pp. 579-590, May 2013.

[4] T. Kleinberger, M. Becker, E. Ras, A. Holzinger, and P. Müller, Ambient Intelligence in Assisted Living: Enable Elderly People to Handle Future Interfaces. Berlin, Heidelberg: Springer Berlin Heidelberg, pp. 103-112, 2007.

[5] M. Danancher, J.-J. Lesage, and L. Litz, "Model-Based Location Tracking of an a priori Unknown Number of Inhabitants in Smart Homes," IEEE Trans. on Automation Science and Engineering, 13(2), pp. 10901101, 2016.

[6] J. Saives, C. Pianon, and G. Faraut, "Activity discovery and detection of behavioral deviations of an inhabitant from binary sensors," IEEE Trans. on Automation Science and Engineering, 12(4), pp. 1211-1224, 2015.

[7] A. A. Chaaraoui, P. Climent-Pérez, and F. FlórezRevuelta, "A review on vision techniques applied to human behaviour analysis for ambient-assisted living," Expert Systems with Applications, 39(12), pp. 1087310888, 2012.

[8] T. V. Duong, H. H. Bui, D. Q. Phung, and S. Venkatesh, "Activity recognition and abnormality detection with the switching hidden semi-markov model," IEEE Computer Society Conference on Computer Vision and Pattern Recognition, 1, pp. 838-845,2005.

[9] T. Van Kasteren, A. Noulas, G. Englebienne, and B. Kröse, "Accurate activity recognition in a home setting," in the ACM tenth international conference on Ubiquitous computing, , pp. 1-9, 2008.

[10] L. Rabiner, "A tutorial on hidden markov models and selected applications in speech recognition," Proceedings of the IEEE, 77(2), pp. 257-286, 1989.

[11] D. J. Cook and N. C. Krishnan, "Activity Learning: Discovering, Recognizing, and Predicting Human Behavior from Sensor Data," John Wiley \& Sons, 2015.

[12] O. D. Lara and M. A. Labrador, "A survey on human activity recognition using wearable sensors," IEEE 
Communications Surveys \& Tutorials, 15(3), pp. 11921209, 2013.

[13] T. L. van Kasteren, G. Englebienne, and B. J. Kröse, "Hierarchical activity recognition using automatically clustered actions," in Springer on Ambient Intelligence, , pp. 82-91,2011.

[14] B. Chikhaoui, S. Wang, and H. Pigot, "A frequent pattern mining approach for adls recognition in smart environments," IEEE International Conference on Advanced Information Networking and Applications, pp. 248-255, 2011.

[15] R. Agrawal and R. Srikant, "Mining sequential patterns," in the IEEE Eleventh International Conference on Data Engineering,, pp. 3-14, 1995.

[16] E. M. Tapia, S. S. Intille, and K. Larson, "Activity recognition in the home using simple and ubiquitous sensors," in Springer on Pervasive Computing, 2004.

[17] A. Fleury, M. Vacher, and N. Noury, "Svm-based multimodal classification of activities of daily living in health smart homes: sensors, algorithms, and first experimental results," IEEE Transactions on Information Technology in Biomedicine, 14(2), pp. 274283, 2010.

[18] N. C. Krishnan and D. J. Cook, "Activity recognition on streaming sensor data," Pervasive and Mobile Computing, 10(B), pp. 138-154, 2014. 